\title{
Molecular Description of a Novel Orientia Species Causing Scrub Typhus in Chile
}

\author{
Katia Abarca, Constanza Martínez-Valdebenito, ${ }^{1}$ Jenniffer Angulo, ${ }^{1}$ Ju Jiang, \\ Christina M. Farris, Allen L. Richards, Gerardo Acosta-Jamett, Thomas Weitzel
}

Scrub typhus is a potentially fatal rickettsiosis caused by Orientia species intracellular bacteria of the genus Orientia. Although considered to be restricted to the Asia Pacific region, scrub typhus has recently been discovered in southern Chile. We analyzed Orientia gene sequences of 16S rRNA (rrs) and 47-kDa ( $h$ trA) from 18 scrub typhus patients from Chile. Sequences were $\geq 99.7 \%$ identical among the samples for both amplified genes. Their diversity was $3.1 \%-3.5 \%$ for $r$ rs and $11.2 \%-11.8 \%$ for htrA compared with 0 . tsusugamushi and $3.0 \%$ for rrs and $14.8 \%$ for htrA compared with Candidatus Orientia chuto. Phylogenetic analyses of both genes grouped the specimens from Chile in a different clade from other Orientia species. Our results indicate that Orientia isolates from Chile constitute a novel species, which, until they are cultivated and fully characterized, we propose to designate as Candidatus Orientia chiloensis, after the Chiloé Archipelago where the pathogen was identified.

Crub typhus is a potentially fatal rickettsial infection transemitted by larval stage trombiculid mites called chiggers. Scrub typhus is caused by Orientia tsutsugamushi, a strictly intracellular bacterium with a remarkable genetic and antigenic diversity $(1,2)$. Although this disease has been known since at least 313 $\mathrm{CE}$ and currently threatens $>1$ billion people in Asia and Australasia, it is widely underdiagnosed and underreported $(3,4)$. Disinterest has been influenced by the perception that scrub typhus is a geographically limited disease, threatening rural populations within

Author affiliations: Escuela de Medicina, Pontificia Universidad Católica de Chile, Santiago, Chile (K. Abarca, C. MartínezValdebenito, J. Angulo); Naval Medical Research Center, Silver Spring, Maryland, USA (J. Jiang, C.M. Farris); Uniformed Services University of the Health Sciences, Bethesda, Maryland, USA (A.L. Richards); Facultad de Ciencias Veterinarias, Universidad Austral de Chile, Valdivia, Chile (G. Acosta-Jamett); Clínica Alemana de Santiago, Facultad de Medicina Clínica Alemana, Universidad del Desarrollo, Santiago (T. Weitzel)

DOI: https://doi.org/10.3201/eid2609.200918 a certain region, known as the "tsutsugamushi triangle," and rarely affecting travelers $(5,6)$.

This paradigm, however, has recently been brought into question with evidence of scrub typhus being found in the Middle East, Africa, and South America (7-12). Genomic information on Orientia strains from Chile has been insufficient and scrub typhus in the Middle East region is caused by a new Orientia species, Candidatus Orientia chuto (6), highlighting that our current knowledge on the spectrum of Orientia species is incomplete (13). Here, we discuss the molecular description and phylogenetic analysis of a potential third pathogenic Orientia species detected in 18 patients with scrub typhus in southern Chile.

\section{Materials and Methods}

\section{Patients and Samples}

The clinical samples described in this study were derived from 18 patients with confirmed scrub typhus diagnosed during February 2016-February 2019. All cases were acquired in southern Chile and diagnosed as part of an ongoing surveillance project of the Chilean Rickettsia and Zoonosis Research Group. The project was approved by the Comité Ético Científico, Pontificia Universidad Católica de Chile (Santiago, Chile; \#12-170 and \#160816007) and the Naval Medical Research Center (Silver Spring, MD, USA; PJT-16-24) $(9,14,15)$.

We collected, stored, and extracted DNA from buffy coat preparations and eschar specimens as described elsewhere $(9,15)$. Eschar samples consisted of swab specimens taken from the base or crust material of eschar, which we mechanically desegregated using sterile glass beads. DNA was automatically extracted from eschar and blood samples using MagNA Pure System (Roche Molecular Systems, https://diagnostics.roche. com), according to the manufacturer's instructions. All included DNA specimens derived from eschar samples,

${ }^{1}$ These authors contributed equally to this article. 
except the BM2016-I sample, which was only from the buffy coat specimen.

\section{PCR Assays and Sequencing}

We initially assessed all extracted DNA samples by a newly designed genus-specific quantitative PCR (Orien16S qPCR assay), as described elsewhere $(15,16)$. For further analysis, we performed seminested PCRs targeting the 16S rRNA gene ( $r r s), 47-\mathrm{kDa}$ high-temperature requirement A gene ( $h t r A)$, and 56-kDa typespecific antigen gene (tsa) to the qPCR Orien16S-positive samples (Appendix Table 1, https:/ / wwwnc.cdc. gov/EID/article/26/9/20-0918-App1.pdf). We sequenced PCR amplicons for both DNA strands using Sanger sequencing method (Psomagen Inc., https:// psomagen.com). Two independent investigators analyzed the chromatogram of each sequence and aligned them using BioEdit version 7.0.5.3 (17) Sequences from these scrub typhus patients in Chile were submitted to GenBank under accession no. MK329247 (rrs), MN231837 (rrs), MT435057 (rrs), MK343091 (htrA), and MT431624 (htrA).

\section{Phylogenetic Analysis}

We compared Orientia DNA sequences from the 2016-2019 scrub typhus patients with DNA from the first Orientia scrub typhus patient in Chile from 2006 and that of distinct Orientia species, including O. tsutsugamushi and Candidatus O. chuto, different Rickettsia species, and other microorganisms retrieved from GenBank and aligned using ClustalW (http://www. clustal.org). We used MEGAX software (https:// www.megasoftware.net) to infer phylogenetic analyses by the maximum-likelihood method (18) and to perform the search for the most appropriate model of nucleotide substitution for phylogenetic analysis according to the Bayesian information criterion. For the maximum-likelihood method, we obtained initial trees for the heuristic search automatically by applying neighbor-joining and Bio neighbor-joining algorithms to a matrix of pairwise distances estimated using the maximum composite likelihood approach and then selecting the topology with superior log likelihood value. We based the support of the topology on a bootstrapping of 1,000 replicates; the positions equivalent to gaps or missing data were deleted.

\section{Comparison of Nucleotide Diversity}

We created consensus sequences of the generated amplicons after alignment in BioEdit version 7.0.5.3, which we compared with respective sequences of $O$. tsutsugamushi and Candidatus $\mathrm{O}$. chuto strains as well as the first Orientia case from Chile, obtained from GenBank.
A sequence identity matrix was constructed in BioEdit version 7.0.5.3. The selected databases and algorithms used for alignment and comparison of sequences were in accordance with current recommendations for the taxonomical characterization of prokaryote strains (19).

\section{Results}

\section{Cases}

The 18 investigated scrub typhus cases were acquired in 3 regions currently known to be endemic for scrub typhus (15), Biobío, Los Lagos, and Aysen, which span $>1,120 \mathrm{~km}$ (latitude $38^{\circ} 03^{\prime} \mathrm{S}$ to $47^{\circ} 47^{\prime} \mathrm{S}$ ) in Chile; 5 of the 18 cases were from Chiloé Island (Los Lagos), where the initial case was reported (Table 1). At the time they sought treatment, all but 1 patient exhibited the 3 clinical signs characteristic of scrub typhus: fever, maculopapular rash, and inoculation eschar. The presence of Orientia genomic DNA was confirmed in all cases by qPCR Orien16S from buffy coat or eschar material (Table 1). All of the patients recovered from scrub typhus without sequelae, 16 after treatment with doxycycline, 1 after treatment with azithromycin, and 1 without specific antimicrobial therapy. Further epidemiologic and clinical details of some of the patients have been published elsewhere $(9,15,20)$.

\section{DNA Sequences and Phylogenetic Analyses}

We successfully amplified fragments of $r$ rs from 18 cases and $h t r A$ from 17 cases; the primers for tsa failed to produce amplicons. For all assays, we successfully amplified a well-defined Orientia strain (Kawasaki clade) from South Korea as a positive control (6). The lengths of clean reads were $886 \mathrm{nt}$ for $\mathrm{rrs}$ and 950 $\mathrm{nt}$ for $h \mathrm{tr} A$. Sequences of the isolates showed a high nucleotide identity $(99.7 \%-100 \%)$ for both genes (Appendix Table 1), with a maximum divergence of 2 nucleotides. We were able to distinguish 3 distinct rrs genotypes (1,2, and 3) and 2 genotypic variants of $h \operatorname{trA}(\mathrm{a}$ and $\mathrm{b}$ ) (Appendix Table 2). HtrA variants, although determined by only 1 nucleotide, led to distinct DNA codons with leucine versus phenylalanine. The genotype 1a samples $(n=10)$ derived from Los Lagos (continental and Chiloé Island), Biobío, and Aysén regions, whereas genotypes $2 b(n=3)$ occurred in the continental Los Lagos region, $3 a(n=1)$ in Chiloé Island, and genotype $3 \mathrm{~b}(\mathrm{n}=3)$ in the Los Lagos region, both continental and Chiloé Island. For 1 genotype 2 strain, we could not amplify $h$ trA (Appendix Table 2). Phylogenetic analyses of both genes from the DNA specimens from Chile formed a unique cluster separate from the 14 O. tsutsugamushi strains included in the analysis as well as from 
Table 1. Epidemiologic, clinical, and diagnostic features of 18 scrub typhus patients, southern Chile, 2016-2019*

\begin{tabular}{|c|c|c|c|c|c|c|c|c|c|c|}
\hline \multirow{2}{*}{$\begin{array}{l}\text { Patient } \\
\text { no. }\end{array}$} & \multirow[b]{2}{*}{ Isolate no. } & \multirow{2}{*}{$\begin{array}{c}\text { Patient } \\
\text { age, y/sex }\end{array}$} & \multicolumn{2}{|c|}{ Exposure } & \multicolumn{3}{|c|}{ Clinical signs } & \multicolumn{2}{|c|}{ qPCR results } & \multirow[b]{2}{*}{ Ref. } \\
\hline & & & Date & Region & Fever & Rash & Eschar & Buffy coat & Eschar & \\
\hline 1 & BM2016-I & $55 / \mathrm{M}$ & $2016 \mathrm{Feb}$ & Los Lagos† & Yes & Yes & Yes & Positive & ND & (9) \\
\hline 2 & LC2016-I & $42 / \mathrm{M}$ & 2016 Feb & Los Lagos† & Yes & No & Yes & ND & Positive & (30) \\
\hline 3 & MS2016-M & 43/M & 2016 Mar & Aysén & Yes & Yes & Yes & Positive & Positive & (15) \\
\hline 4 & IS2017-M & 56/M & 2017 Feb & Los Lagos & Yes & Yes & Yes & Positive & Positive & (15) \\
\hline 5 & NV2017-I & 73/M & 2017 Feb & Los Lagos† & Yes & Yes & Yes & ND & Positive & (30) \\
\hline 6 & AE2018-M & $25 / M$ & 2018 Feb & Los Lagos & Yes & Yes & Yes & ND & Positive & (15) \\
\hline 7 & FC2018-M & $22 / F$ & 2018 Feb & Los Lagos & Yes & Yes & Yes & Positive & Positive & (15) \\
\hline 8 & AF2018-M & $39 / M$ & 2018 Feb & Los Lagos & Yes & Yes & Yes & Positive & Positive & (15) \\
\hline 9 & EC2018-M & 28/M & 2018 Feb & Biobío & Yes & Yes & Yes & Positive & Positive & (15) \\
\hline 10 & VP2018-M & $21 / F$ & 2018 Mar & Los Lagos & Yes & Yes & Yes & Positive & Positive & (15) \\
\hline 11 & SH2018-M & $49 / F$ & 2018 Dec & Los Lagos & Yes & Yes & Yes & Negative & Positive & (30) \\
\hline 12 & GM2019-I & $30 / F$ & 2019 Feb & Los Lagos† & Yes & Yes & Yes & ND & Positive & (30) \\
\hline 13 & JC2019-I & 54/M & 2019 Feb & Los Lagos† & Yes & Yes & Yes & ND & Positive & (30) \\
\hline 14 & CC2019-M & 63/M & 2019 Feb & Los Lagos & Yes & Yes & Yes & ND & Positive & (30) \\
\hline 15 & CV2019-M & $23 / F$ & 2019 Feb & Los Lagos & Yes & Yes & Yes & ND & Positive & (30) \\
\hline 16 & MA2019-M & 53/M & 2019 Feb & Los Lagos & Yes & Yes & Yes & ND & Positive & (30) \\
\hline 17 & MV2019-M & $54 / F$ & 2019 Feb & Los Lagos & Yes & Yes & Yes & ND & Positive & (30) \\
\hline 18 & SG2019-M & $41 / \mathrm{M}$ & 2019 Feb & Los Lagos & Yes & Yes & Yes & ND & Positive & (30) \\
\hline
\end{tabular}

Candidatus O. chuto (Figures 1,2). However, the rrs sequence from the 2016-2019 samples grouped together with that from the first scrub typhus case in Chile (Figure 1) (8).

Details of discrepancies in the nucleotide sequences were evaluated by identity matrices. For $r$ s from the isolates from Chile, identity of the consensus sequence ranged from $96.5 \%$ to $97.0 \%$ compared with $O$. tsutsugamushi and Candidatus O. chuto (Table 2, https://wwwnc.cdc.gov/EID/article/26/9/200918-T2.htm). A higher diversity was observed for htrA, with sequence identity of $88.2 \%-88.8 \%$ compared with O. tsutsugamushi and $85.2 \%$ with Candidatus O. chuto (Table 3, https://wwwnc.cdc.gov/ EID/article/26/9/20-0918-T3.htm). We observed a GTA insertion (valine) in position 28 of $h t r A$ in all 18 samples, similar to Candidatus O. chuto, but this substitution was not observed in O. tsutsugamushi strains.

\section{Species Designation}

The $r$ rs sequences we analyzed showed a divergence of $\geq 3 \%$ from known Orientia species, indicating that the isolates from Chile constitute a novel species within the genus Orientia (family Rickettsiaceae, order Rickettsiales, class Alphaproteobacteria). Our designation of the bacteria as a new species was corroborated by the divergence of $h t r A$ and our inability to generate amplicons with primers of $O$. tsutsugamushi type-specific antigen gene tsa. Until a type strain is cultivated and characterized, we propose the designation Candidatus Orientia chiloensis for the novel species, after the Chiloé Archipelago (Los Lagos Region, Chile) where the pathogen was first identified $(8,9)$.

\section{Discussion}

Because of new diagnostic tools and increasing clinical awareness, our knowledge of rickettsial infections has increased over recent decades $(23,24)$. For scrub typhus, which has been considered the most important rickettsiosis in Asia and Australasia, the discovery of new endemic regions outside of the traditional tsutsugamushi triangle raises questions about established paradigms (25). Since 2006, multiple patients with scrub typhus have been reported in southern Chile, $>12,000 \mathrm{~km}$ away from known endemic regions $(8,9,15)$. In addition, a case of scrub typhus caused by a novel species, Candidatus O. chuto, was diagnosed on the Arabian Peninsula (7). These findings, together with serologic and molecular data from sub-Saharan Africa and Europe, suggest that scrub typhus caused by various Orientia species might have a much wider than previously known, possibly global, distribution $(4,26,27)$.

Most clinicoepidemiologic and ecologic aspects of scrub typhus in South America are currently unknown. A recent study on Chiloé Island suggested that trombiculid mites of the genus Herpetacarus, which were found to be infected with Orientia-species bacteria, might serve as vectors (28); preliminary phylogenetic analyses showed that the mite-associated strains were $99 \%-100 \%$ identical to those from patients (29). Clinically, the $>40$ patients with scrub typhus diagnosed in southern Chile during 2015-2019 sought treatment for conditions similar to those for scrub typhus from the Asia Pacific region-fever, generalized rash, and inoculation eschar-and, similarly, had a rapid response to treatment with tetracycline or azithromycin (30). Early molecular and 
serologic data suggest that the Orientia species in Chile diverge from those in the Asia-Pacific region $(8,9,15)$, but whether they represent distinct $O$. tsutsugamushi strains or a new species remained inconclusive. Our phylogenetic analyses of larger DNA segments from 2 conserved genes support the conclusion that the

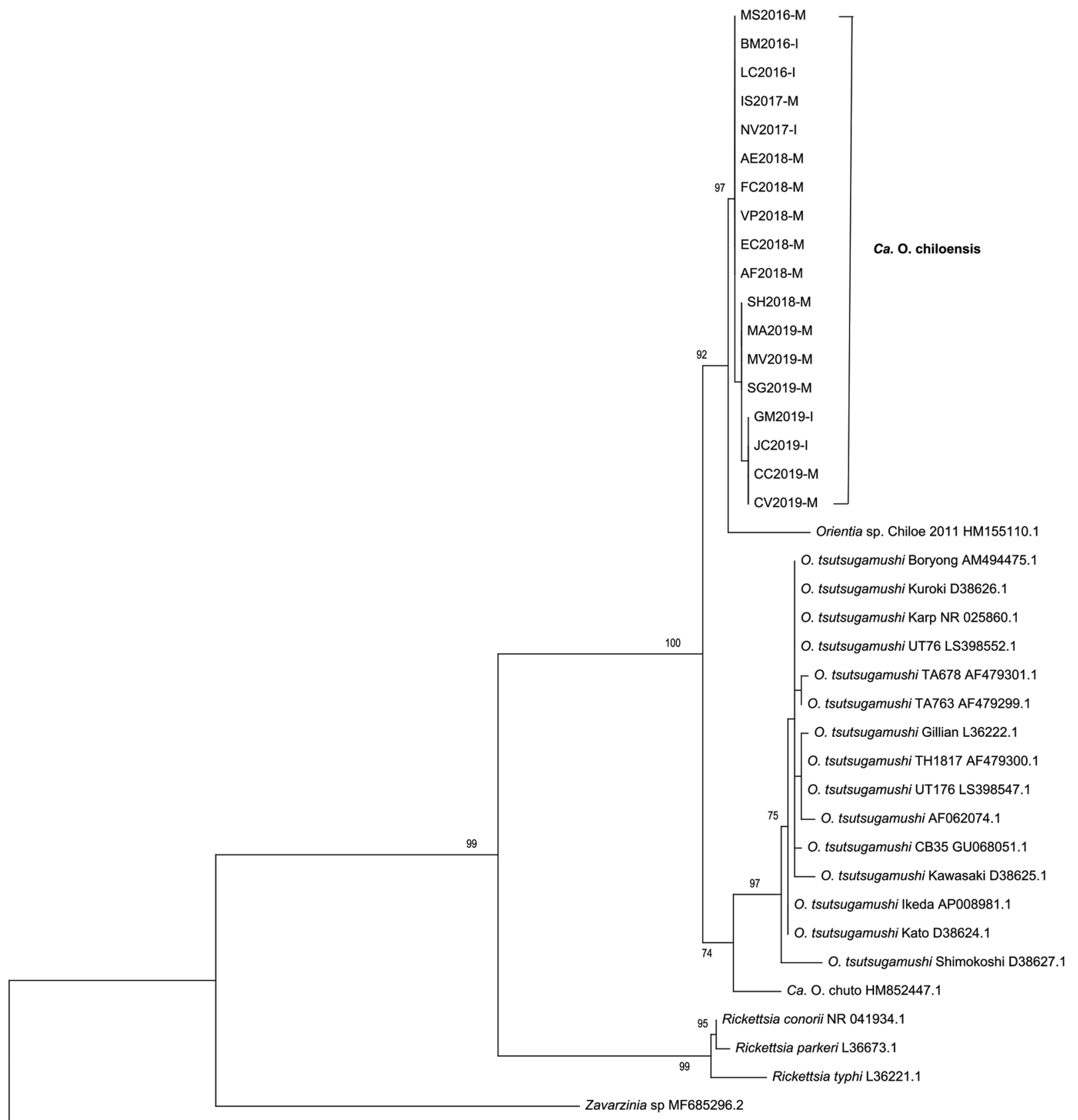

Paraburkholderia bannensis MK041544.1

0.020

Figure 1. Phylogenetic analyses of sequences of the 16S rRNA gene ( $r r s)$ from scrub typhus cases in Chile compared with those from different Orientia and Rickettsia species and other microorganisms. We inferred the evolutionary history by using the maximumlikelihood method based on the Kimura 2-parameter model (21), according to the Bayesian information criterion for these sequences. The analysis involved $39 \mathrm{nt}$ sequences and a total of 875 positions in the final dataset. The trees is drawn to scale, with branch lengths measured in the number of substitutions per site. All positions containing gaps and missing data were eliminated. For isolates from the cases in this study, the suffix "M" indicates an origin in mainland Chile and "l" an origin on Chiloé Island; these isolates clustered into a proposed new species provisionally named Candidatus Orientia chiloensis. GenBank accession numbers are indicated for reference sequences. Scale bar indicates nucleotide divergence. 
isolates from patients in Chile cluster outside known Orientia species and represent a distinct species.

Culture-independent sequencing techniques play an important role in prokaryotic taxonomy, especially for strictly intracellular bacteria $(31,32)$. For the description of new species, sequence analyses of the $16 \mathrm{~S}$ rRNA gene $(r r s)$ are paramount. $\mathrm{A} \geq 3 \%$ divergence of $r$ rs sequences from those in known species is the accepted threshold suggesting a novel species
(19), although corrected levels of $\geq 1.30 \%-1.35 \%$ have been suggested $(33,34)$. Isolates with $r r s$ sequence differences of $>5 \%-6 \%$ might belong to a distinct genus, if they display unique phenotypic differences (35). Distinct, lower thresholds have been developed for Rickettsia spp. (31), but this approach remains controversial among rickettsiologists (36). As should be the case for all molecularly defined novel species and genera, we have classified this proposed species as

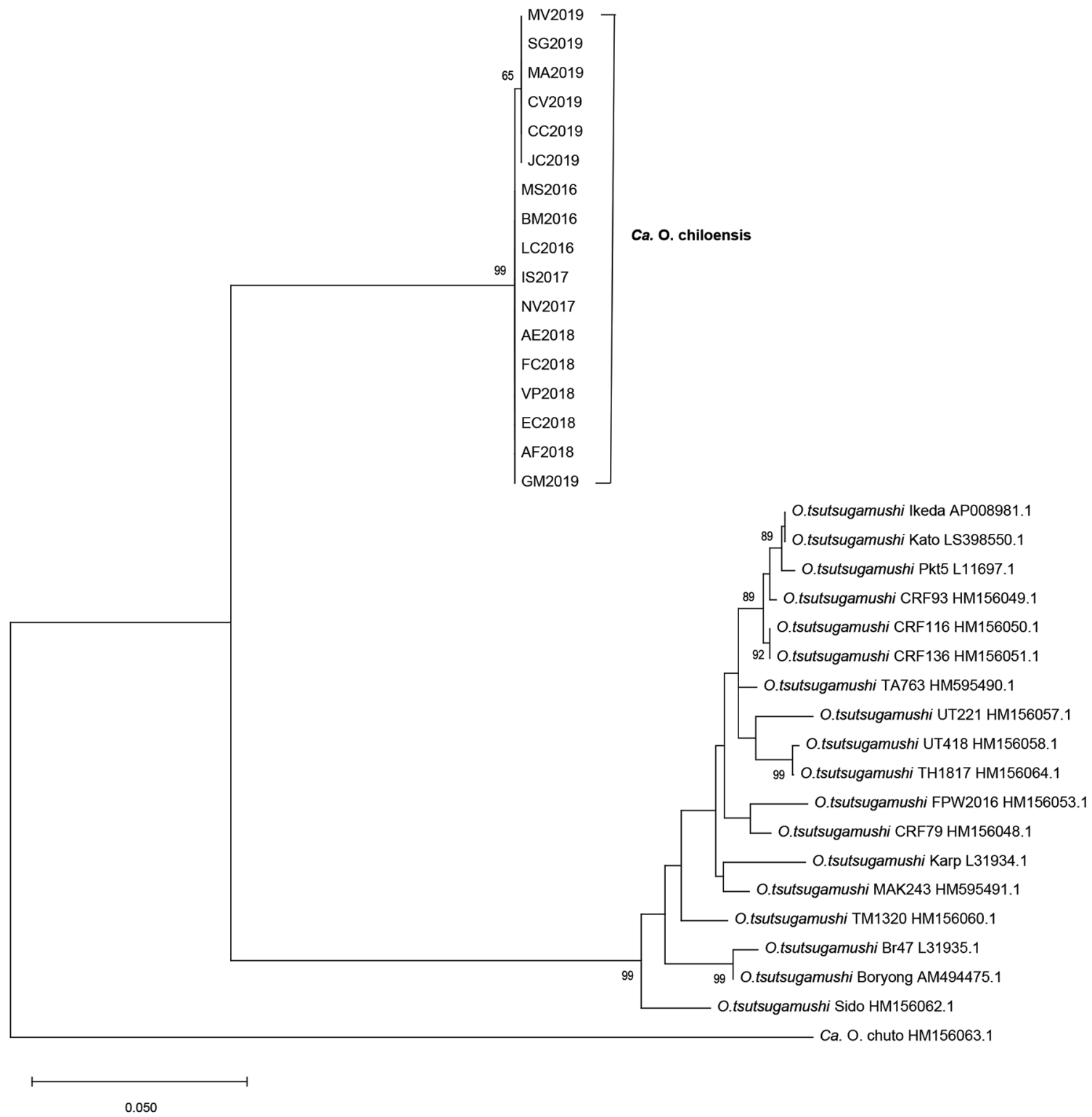

Figure 2. Phylogenetic analyses of sequences of the 47-kDa gene ( $h$ trA) from scrub typhus cases from Chile in comparison to different Orientia species. For the phylogenetic tree, the maximum-likelihood method based on the Hasegawa-Kishino-Yano model was applied (22). A discrete gamma distribution was used to model evolutionary rates differences among sites. The analysis involved 37 nucleotide sequences and a total of 736 positions in the final dataset. The tree is drawn to scale, with branch lengths measured in the number of substitutions per site. All positions containing gaps and missing data were eliminated. Isolates from the cases in this study clustered into a proposed new species provisionally named Candidatus Orientia chiloensis. GenBank accession numbers are indicated for reference sequences. Scale bar indicates nucleotide divergence. 
Novel Orientia Species and Scrub Typhus in Chile

Table 2. Identity matrix of $16 \mathrm{~S}$ rRNA gene ( $r$ rs) showing percentage of pairwise identity of 886 nt consensus sequence of 18 recent Candidatus Orientia chiloensis cases in Chile with the first Orientia case from Chile in 2006, 16 O. tsutsugamushi strains, and Candidatus Orientia chuto

\begin{tabular}{|c|c|c|c|c|c|c|c|c|c|c|c|c|c|c|c|c|c|}
\hline Sequences ${ }^{*} \dagger$ & 1 & 2 & 3 & 4 & 5 & 6 & 7 & 8 & 9 & 10 & 11 & 12 & 13 & 14 & 15 & 16 & 17 \\
\hline \multicolumn{18}{|l|}{1 Chiloensis } \\
\hline 3 Chuto & 97.0 & 95.8 & & & & & & & & & & & & & & & \\
\hline 5 Kuroki & 96.7 & 95.7 & 97.7 & 100 & & & & & & & & & & & & & \\
\hline 6 Gilliam & 96.5 & 95.4 & 97.5 & 99.7 & 99.7 & & & & & & & & & & & & \\
\hline 7 Karp & 96.8 & 95.8 & 97.8 & 99.8 & 99.8 & 99.6 & & & & & & & & & & & \\
\hline 8 Kawasaki & 96.7 & 95.7 & 97.1 & 99.2 & 99.2 & 99.2 & 99.3 & & & & & & & & & & \\
\hline 11 Shimokoshi & 96.9 & 96.1 & 96.9 & 98.4 & 98.4 & 98.4 & 98.5 & 98.6 & 98.6 & 98.6 & & & & & & & \\
\hline 12 CB35 & 96.7 & 95.7 & 97.9 & 99.7 & 99.7 & 99.5 & 99.8 & 99.2 & 99.7 & 99.7 & 98.4 & & & & & & \\
\hline 13 TA678 & 96.6 & 95.6 & 97.6 & 99.6 & 99.6 & 99.4 & 99.7 & 99.0 & 99.6 & 99.6 & 98.3 & 99.6 & & & & & \\
\hline 14 TA763 & 96.5 & 95.4 & 97.5 & 99.5 & 99.5 & 99.3 & 99.6 & 98.9 & 99.5 & 99.5 & 98.1 & 99.5 & 99.6 & & & & \\
\hline $15 \mathrm{TH} 1817$ & 96.5 & 95.6 & 97.5 & 99.5 & 99.5 & 99.5 & 99.6 & 98.9 & 99.5 & 99.5 & 98.1 & 99.5 & 99.4 & 99.3 & & & \\
\hline 16 UT176 & 96.8 & 95.8 & 97.6 & 99.6 & 99.6 & 99.6 & 99.7 & 99.3 & 99.6 & 99.6 & 98.5 & 99.6 & 99.5 & 99.4 & 99.6 & & \\
\hline
\end{tabular}

Candidatus, until type strains can be cultivated and fully described (37).

The novel Orientia species presented here fulfills the rrs gene criteria described in the previous sections. Our designation of a novel species was affirmed by a high divergence of another genomic marker, $h t r A$, which diverged $>11 \%$ from $O$. tsutsugamushi and of $>14 \%$ from Candidatus O. chuto (Table 3). This con- served gene diverges only $<3.7 \%$ among 0 . tsutsugamushi isolates (38). The O. tsutsugamushi type-specific antigen gene, $t s a$, which has a much higher diversity than $h \operatorname{tr} A$ (1), was not amplifiable from isolates from Chile using primers designed for O. tsutsugamushi. This suggests that the Candidatus $\mathrm{O}$. chiloensis tsa is unique, requiring the assessment of additional primers, possibly based on results from a future WGS.

Table 3. Identity matrix of 47-kDa high temperature requirement A gene (htrA) showing percentage of pairwise identity of $950 \mathrm{nt}$ consensus sequence of 17 Candidatus Orientia chiloensis cases from Chile with 18 strains of $O$. tsutsugamushi and Candidatus Orientia chuto

\begin{tabular}{|c|c|c|c|c|c|c|c|c|c|c|c|c|c|c|c|c|c|c|c|}
\hline Sequences $^{*} \dagger$ & 1 & 2 & 3 & 4 & 5 & 6 & 7 & 8 & 9 & 10 & 11 & 12 & 13 & 14 & 15 & 16 & 17 & 18 & 19 \\
\hline \multicolumn{20}{|l|}{1 Chiloensis } \\
\hline 3 lkeda & 88.5 & 83.7 & & & & & & & & & & & & & & & & & \\
\hline $5 \mathrm{Kp} 47$ & 88.2 & 83.7 & 97.5 & 97.3 & & & & & & & & & & & & & & & \\
\hline $6 \mathrm{Br} 47$ & 88.3 & 83.8 & 96.9 & 96.7 & 96.5 & & & & & & & & & & & & & & \\
\hline 7 Boryong & 88.4 & 83.7 & 97.2 & 97.0 & 96.8 & 99.4 & & & & & & & & & & & & & \\
\hline 8 CRF116 & 88.3 & 83.5 & 99.5 & 99.3 & 97.5 & 96.9 & 97.2 & & & & & & & & & & & & \\
\hline 11 MAK243 & 88.8 & 84.2 & 98.5 & 98.3 & 98.0 & 97.5 & 97.8 & 98.5 & 98.0 & 98.5 & & & & & & & & & \\
\hline 12 CRF79 & 88.3 & 83.7 & 98.7 & 98.5 & 97.7 & 96.9 & 97.2 & 98.7 & 98.7 & 98.7 & 98.5 & & & & & & & & \\
\hline 13 TM1320 & 88.6 & 83.4 & 98.4 & 98.2 & 97.0 & 97.4 & 97.7 & 98.2 & 97.8 & 98.4 & 97.9 & 97.6 & & & & & & & \\
\hline 14 UT221 & 88.8 & 84.1 & 98.5 & 98.3 & 96.9 & 97.1 & 97.3 & 98.5 & 97.4 & 98.5 & 98.2 & 98.0 & 97.5 & & & & & & \\
\hline 15 UT418 & 88.4 & 84.1 & 98.5 & 98.3 & 97.5 & 96.9 & 97.2 & 98.5 & 97.5 & 98.5 & 98.2 & 98.4 & 97.4 & 98.4 & & & & & \\
\hline 16 Sido & 88.7 & 83.6 & 96.9 & 96.7 & 96.0 & 96.7 & 97.2 & 96.7 & 97.3 & 96.9 & 97.2 & 96.7 & 97.2 & 96.9 & 96.3 & & & & \\
\hline $\begin{array}{l}{ }^{*} \text { All sequence } \\
\text { Orientia chuto } \\
\text { †Numbers in } \\
\text { GenBank acc } \\
10 \text {, LS398550 }\end{array}$ & strains & 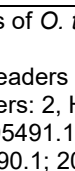 & . & & & 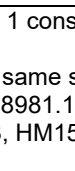 & 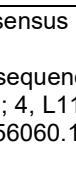 & 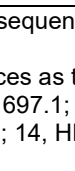 & 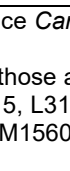 & 4.1 & L3 & 5.1 & 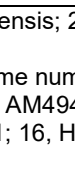 & 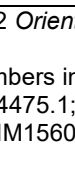 & $8, \mathrm{H}$ & 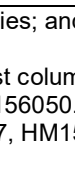 & d $3 \mathrm{C}$ & & 3.1 \\
\hline
\end{tabular}


Currently, no Orientia culture isolate from Chile is available.

Surprisingly, the rrs and $h$ trA sequences from the 18 Orientia samples from Chile were almost identical, showing a maximum variability of only 2 nucleotides. This genetic homogeneity over a wide geographic range is in sharp contrast to O. tsutsugamushi (38). As a unique characteristic among obligate intracellular pathogens, this species displays a dramatic genomic and phenotypic heterogeneity (1), which might be related to homologous recombination and lateral gene transfer (39). Among O. tsutsugamushi isolates, for example, the divergence of reported $r r s$ sequences are up to $1.5 \%$ and for $h$ trA sequences up to $3.6 \%(1,38)$, compared with $\leq 0.3 \%$ observed among isolates from Orientia DNA from Chile.

The most frequently applied phenotypic and molecular marker of $O$. tsutsugamushi strain heterogeneity is the highly variable 56-kDa TSA. This Orientia-specific surface protein is also known to be an important determinant of strain-specific pathogenicity and immunity (1). As we mentioned, we were not able to generate amplicons of strains from Chile with the applied tsa PCR or with tsa qPCR (41) or other commonly used primers (e.g., r56_2057). In a previous report, short tsa sequences were produced from 2 samples, but only after prolonged amplification cycles (9). These findings strongly suggest that tsa of Candidatus $\mathrm{O}$. chiloensis is highly divergent from those of other Orientia species. Becaue the TSA surface protein is the main antigenic determinant, such divergence might explain the low serologic cross-reactivity, which was observed in patients with scrub typhus and in seroprevalence studies in Chile using O. tsutsugamushi whole-cell or recombinant antigens $(9,41)$.

In conclusion, our results indicate that scrub typhus in Chile is caused by a novel Orientia species, suggesting an ancient origin of the disease in South America, rather than recent introduction. However, after obtaining cultured isolates of Candidatus $\mathrm{O}$. chiloensis and larger gene sequences including from WGS, deeper comparative studies of the 3 Orientia species and their vectors are necessary to understand the ecology and evolution of these emerging intracellular pathogens, including the mechanisms responsible for the differences in strain variability and surface proteins.

\section{Acknowledgments}

We acknowledge Katia Velasquez and all other physicians who participated in identifying the scrub typhus cases included in this study. We also thank Teresa Azócar and Romina Alarcón for their technical help in processing the clinical samples.
This work was supported by a grant from the Fondo Nacional de Desarrollo Científico y Tecnológico (FONDECYT N ${ }^{\circ} 1130817$ and $\mathrm{N}^{\circ} 1170810$ ) and the Armed Forces Health Surveillance Branch and its Global Emerging Infections Surveillance and Response (GEIS) Section (funding year 2018, ProMIS ID P0017_19_NM_02 NMRC work unit number A0047).

The views expressed in this article reflect the results of research conducted by the authors and do not necessarily reflect the official policy or position of the Department of the Navy, Department of Defense, or the United States Government.

\section{About the Author}

Dr. Abarca is a pediatric infectious diseases specialist and professor at the School of Medicine, Pontificia Universidad Católica de Chile in Santiago, Chile. Her main research interests include vectorborne zoonoses and rickettsial infections as well as vaccine development.

\section{References}

1. Kelly DJ, Fuerst PA, Ching WM, Richards AL. Scrub typhus: the geographic distribution of phenotypic and genotypic variants of Orientia tsutsugamushi. Clin Infect Dis. 2009; 48(Suppl 3):S203-30. https:/ / doi.org/10.1086/ 596576

2. Kim G, Ha NY, Min CK, Kim HI, Yen NT, Lee KH, et al. Diversification of Orientia tsutsugamushi genotypes by intragenic recombination and their potential expansion in endemic areas. PLoS Negl Trop Dis. 2017;11:e0005408. https://doi.org/10.1371/journal.pntd.0005408

3. Paris DH, Shelite TR, Day NP, Walker DH. Unresolved problems related to scrub typhus: a seriously neglected life-threatening disease. Am J Trop Med Hyg. 2013;89:301-7. https://doi.org/10.4269/ajtmh.13-0064

4. Xu G, Walker DH, Jupiter D, Melby PC, Arcari CM. A review of the global epidemiology of scrub typhus. PLoS Negl Trop Dis. 2017;11:e0006062. https://doi.org/10.1371/ journal.pntd.0006062

5. Bonell A, Lubell Y, Newton PN, Crump JA, Paris DH. Estimating the burden of scrub typhus: a systematic review. PLoS Negl Trop Dis. 2017;11:e005838. https://doi.org/10.1371/journal.pntd.0005838

6. Weitzel T, Aylwin M, Martínez-Valdebenito C, Jiang J, Munita JM, Thompson L, et al. Imported scrub typhus: first case in South America and review of the literature. Trop Dis Travel Med Vaccines. 2018;4:10. https:/ / doi.org/10.1186/ s40794-018-0070-8

7. Izzard L, Fuller A, Blacksell SD, Paris DH, Richards AL, Aukkanit N, et al. Isolation of a novel Orientia species ( $O$. chuto sp. nov.) from a patient infected in Dubai. J Clin Microbiol. 2010;48:4404-9. https://doi.org/10.1128/ JCM.01526-10

8. Balcells ME, Rabagliati R, García P, Poggi H, Oddó D, Concha M, et al. Endemic scrub typhus-like illness, Chile. Emerg Infect Dis. 2011;17:1659-63. https:/ / doi.org/10.3201/ eid1709.100960

9. Weitzel T, Dittrich S, López J, Phuklia W, Martinez-Valdebenito C, Velásquez K, et al. Endemic scrub 
typhus in South America. N Engl J Med. 2016;375:954-61. https:/ / doi.org/10.1056/NEJMoa1603657

10. Maina AN, Farris CM, Odhiambo A, Jiang J, Laktabai J, Armstrong J, et al. Q fever, scrub typhus, and rickettsial diseases in children, 2011-2012 Kenya. Emerg Infect Dis. 2016;22:883-6. https://doi.org/10.3201/eid2205.150953

11. Horton KC, Jiang J, Maina A, Dueger E, Zayed A, Ahmed AA, et al. Evidence of Rickettsia and Orientia infections among abattoir workers in Djibouti. Am J Trop Med Hyg. 2016;95:462-5. https://doi.org/10.4269/ ajtmh.15-0775

12. Kocher C, Jiang J, Morrison AC, Castillo R, Leguia M, Loyola S, et al. Scrub typhus in the Peruvian Amazon. Emerg Infect Dis. 2017;23:1389-91. https:/ / doi.org/10.3201/ eid2308.170050

13. Luce-Fedrow A, Lehman ML, Kelly DJ, Mullins K, Maina AN, Stewart RL, et al. A review of scrub typhus (Orientia tsutsugamushi and related organisms): then, now, and tomorrow. Trop Med Infect Dis. 2018;3:pii E8. https://doi.org/10.3390/tropicalmed3010008

14. Abarca K, Weitzel T, Martínez-Valdebenito C, Acosta-Jamett G. Scrub typhus, an emerging infectious disease in Chile [in Spanish]. Rev Chilena Infectol. 2018; 35:696-9. https:// doi.org/10.4067/S0716-10182018000600696

15. Weitzel T, Martínez-Valdebenito C, Acosta-Jamett G, Jiang J, Richards AL, Abarca K. Scrub typhus in continental Chile, 2016-2018. Emerg Infect Dis. 2019;25:1214-7. https:// doi.org/10.3201/eid2506.181860

16. Jiang J, Martínez-Valdebenito C, Weitzel T, Abarca K, Richards AL. Development of an Orientia genus-specific quantitative real-time PCR assay and the detection of Orientia species in DNA preparations from O. tsutsugamushi, Candidatus Orientia chuto, and Orientia species from Chile. In: Abstracts of the 29th Meeting of the American Society for Rickettsiology; Milwaukee, WI, USA; 2018 Jun 16-19. Abstract no. 46.

17. Hall TA. Bioedit: a user-friendly biological sequence alignment editor and analysis program for Windows 95/98/ NT. Nucleic Acids Symp Ser. 1999;41:95-8.

18. Kumar S, Stecher G, Li M, Knyaz C, Tamura K. MEGA X: molecular evolutionary genetics analysis across computing platforms. Mol Biol Evol. 2018;35:1547-9. https://doi.org/ 10.1093/molbev/msy096

19. Tindall BJ, Rosselló-Móra R, Busse HJ, Ludwig W, Kämpfer P. Notes on the characterization of prokaryote strains for taxonomic purposes. Int J Syst Evol Microbiol. 2010;60:249-66. https:/ / doi.org/10.1099/ijs.0.016949-0

20. Weitzel T, Acosta-Jamett G, Martínez-Valdebenito C, Richards AL, Grobusch MP, Abarca K. Scrub typhus risk in travelers to southern Chile. Travel Med Infect Dis. 2019;29:78-9. https:// doi.org/10.1016/j.tmaid.2019.01.004

21. Kimura M. A simple method for estimating evolutionary rates of base substitutions through comparative studies of nucleotide sequences. J Mol Evol. 1980;16:111-20. https://doi.org/10.1007/BF01731581

22. Hasegawa M, Kishino H, Yano T. Dating of the human-ape splitting by a molecular clock of mitochondrial DNA. J Mol Evol. 1985;22:160-74. https://doi.org/10.1007/BF02101694

23. Merhej V, Angelakis E, Socolovschi C, Raoult D. Genotyping, evolution and epidemiological findings of Rickettsia species. Infect Genet Evol. 2014;25:122-37. https:/ / doi.org/10.1016/ j.meegid.2014.03.014

24. Richards AL. Worldwide detection and identification of new and old rickettsiae and rickettsial diseases. FEMS Immunol Med Microbiol. 2012;64:107-10. https://doi.org/10.1111/ j.1574-695X.2011.00875.x
25. Walker DH. Scrub typhus - scientific neglect, everwidening impact. N Engl J Med. 2016;375:913-5. https:// doi.org/10.1056/NEJMp1608499

26. Jiang J, Richards AL. Scrub typhus: no longer restricted to the tsutsugamushi triangle. Trop Med Infect Dis. 2018;3:pii E11. PubMed https://doi.org/10.3390/ tropicalmed3010011

27. Elliott I, Pearson I, Dahal P, Thomas NV, Roberts T, Newton PN. Scrub typhus ecology: a systematic review of Orientia in vectors and hosts. Parasit Vectors. 2019;12:513. https://doi.org/10.1186/s13071-019-3751-x

28. Acosta-Jamett G, Martínez-Valdebenito C, Beltrami E, Silva-de La Fuente MC, Jiang J, Richards AL, et al. Identification of trombiculid mites (Acari: Trombiculidae) on rodents from Chiloé Island and molecular evidence of infection with Orientia species. PLoS Negl Trop Dis. 2020;14:e0007619. https://doi.org/10.1371/journal. pntd.0007619

29. Martínez-Valdebenito C, Silva-de la Fuente MC, Acosta-Jamett G, Weitzel T, Jiang J, Richards AL, Abarca K. Molecular detection of Orientia spp. in trombiculid mites collected from rodents on Chiloé Island, Chile. In: Conference Book of the 2nd Asia Pacific Rickettsia Conference; Chiang Rai, Thailand; 2019 Nov 3-6. Abstract no. 33. p. 66.

30. Abarca K, Kuijpers S, Velásquez K, Martínez-Valdebenito C, Acosta-Jamett G, Weitzel T. Demographic, clinical, and laboratory features of South American scrub typhus in southern Chile, 2015-2019. In: Conference Book of the 2nd Asia Pacific Rickettsia Conference; Chiang Rai, Thailand; 2019 Nov 3-6. Abstract no. 26. p. 33.

31. Fournier PE, Raoult D. Current knowledge on phylogeny and taxonomy of Rickettsia spp. Ann N Y Acad Sci. 2009;1166:1-11. https://doi.org/10.1111/j.1749-6632. 2009.04528.x

32. Parks DH, Chuvochina M, Waite DW, Rinke C, Skarshewski A, Chaumeil PA, et al. A standardized bacterial taxonomy based on genome phylogeny substantially revises the tree of life. Nat Biotechnol. 2018;36:996-1004. https://doi.org/10.1038/nbt.4229

33. Stackebrandt E, Ebers J. Taxonomic parameters revisited: tarnished gold standards. Microbiol Today. 2006;33:152-5.

34. Kim M, Oh HS, Park SC, Chun J. Towards a taxonomic coherence between average nucleotide identity and $16 \mathrm{~S}$ rRNA gene sequence similarity for species demarcation of prokaryotes. Int J Syst Evol Microbiol. 2014;64:346-51. https://doi.org/10.1099/ijs.0.059774-0

35. Qin QL, Xie BB, Zhang XY, Chen XL, Zhou BC, Zhou J, et al. A proposed genus boundary for the prokaryotes based on genomic insights. J Bacteriol. 2014;196:2210-5. https://doi.org/10.1128/JB.01688-14

36. Walker DH. Rickettsiae and rickettsial infections: the current state of knowledge. Clin Infect Dis. 2007;45(Suppl 1):S39-44. https:/ / doi.org/10.1086/518145

37. Raoult D, Fournier PE, Eremeeva M, Graves S, Kelly PJ, Oteo JA, et al. Naming of rickettsiae and rickettsial diseases. Ann N Y Acad Sci. 2005;1063:1-12. https:/ / doi.org/10.1196/ annals.1355.002

38. Jiang J, Paris DH, Blacksell SD, Aukkanit N, Newton PN, Phetsouvanh R, et al. Diversity of the 47-kD HtrA nucleic acid and translated amino acid sequences from 17 recent human isolates of Orientia. Vector Borne Zoonotic Dis. 2013;13:367-75. https://doi.org/10.1089/vbz.2012.1112

39. Fleshman A, Mullins K, Sahl J, Hepp C, Nieto N, Wiggins $\mathrm{K}$, et al. Comparative pan-genomic analyses of Orientia tsutsugamushi reveal an exceptional model of 
bacterial evolution driving genomic diversity.

2018;4:e000199. https://doi.org/10.1099/mgen.0.000199

40. Jiang J, Chan TC, Temenak JJ, Dasch GA, Ching WM, Richards AL. Development of a quantitative real-time polymerase chain reaction assay specific for Orientia tsutsugamushi. Am J Trop Med Hyg. 2004;70:351-6. https:/ / doi.org/10.4269/ajtmh.2004.70.351

41. Weitzel T, Acosta-Jametta, G, Jiang J, Martínez-Valdebenito C, Farris C, Richards AL, et al. Human seroepidemiology of Rickettsia and Orientia species in Chile-a cross-sectional study in 5 regions. Ticks Tick Borne Dis. 2020;11:101503.

https:// doi.org/10.1016/j.ttbdis.2020.101503

Address for correspondence: Thomas Weitzel, Laboratorio Clínico, Clínica Alemana de Santiago, Av. Vitacura 5951, Santiago, Chile; email: thomas.weitzel@gmail.com; and Katia Abarca, Departamento de Enfermedades Infecciosas e Inmunología Pediátricas, Pontificia Universidad Católica de Chile, Santiago, Chile; email: katia@med.puc.cl

\title{
etymologia
}

\author{
Aline E. Santana, Fábio P. Sellera
}

\section{Dermatophyte [dur'mə-to-fit"]}

$\mathrm{F}^{\mathrm{t}}$ rom the Greek derma (skin) + phyton (plant), dermatophytes are a group of 3 genera of filamentous fungi (Microsporum, Epidermophyton, and Trichophyton) that have the ability to invade keratinized tissues and cause superficial infections in humans and animals. Dermatophytes were improperly assigned to the Plantae kingdom until 1969, when they were then classified into the Fungi kingdom.

Dermatophytosis is also referred to as ringworm or tinea (Latin for "worm") because it can cause ring-shaped patches that are usually red, itchy, and have worm-like borders. In 1910, Raymond Jacques Adrien Sabouraud, a French dermatologist, was the first to report the morphologic characteristics of dermatophytes. During the decades that followed, taxonomy of dermatophytes has gone through revolutionary changes, mostly due to the advent of molecular diagnosis. Although studies performed in the 21st century have resulted in further classification changes and

\section{Sources}

1. Borman AM, Summerbell RC. Trichophyton,

Microsporum, Epidermophyton, and agents of superficial mycoses. In: Carroll KC, Pfaller MA, Landry ML, McAdam A, Patel R, Richter SS, et al., editors. Manual of clinical microbiology. Vol. 2, 12th ed. Washington: ASM Press; 2019. p. 2208-33.

2. de Hoog GS, Dukik K, Monod M, Packeu A, Stubbe D, Hendrickx M, et al. Toward a novel multilocus consolidation of new species, debates regarding the taxonomy of dermatophyte agents persist.

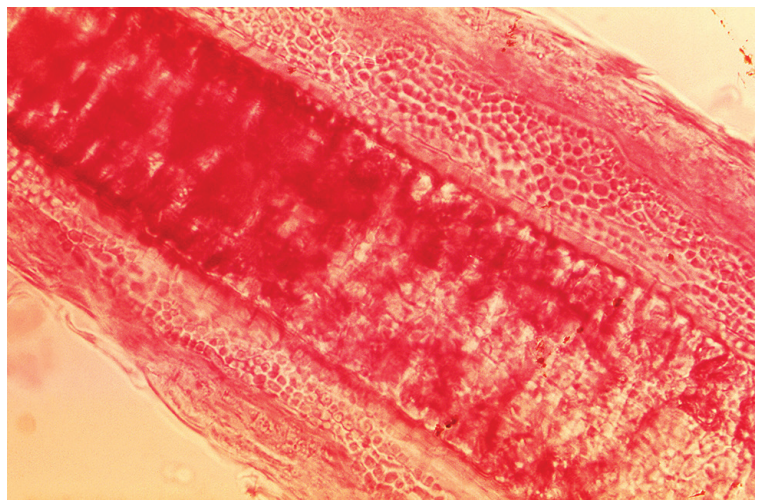

Figure. Photomicrograph of a guinea pig hair shaft specimen revealed ultrastructural features exhibited at the site of a ringworm infection by the dermatophyte, Trichophyton mentagrophytes. Note that the sporangia were confined to the outer region of the hair shaft, known as an exothrix infection. Original magnification $\times 430$. CDC/Dr. Lucille K. Georg, 1968.

phylogenetic taxonomy for the dermatophytes. Mycopathologia. 2017;182:5-31. https:/ / doi.org/ 10.1007/s11046-016-0073-9

3. Sabouraud R. The moths [in French]. Paris: Masson; 1910.

4. Whittaker RH. New concepts of kingdoms or organisms. Evolutionary relations are better represented by new classifications than by the traditional two kingdoms. Science. 1969;163:150-60. https:// doi.org/10.1126/science.163.3863.150

Author affiliation: Universidade de São Paulo, São Paulo, Brazil

Address for correspondence: Fábio P. Sellera, Department of Internal Medicine, School of Veterinary Medicine and Animal Science, Universidade de São Paulo, São Paulo SP- 05508-270, Brazil; email: fsellera@usp.br

DOI: https://doi.org/10.3201/eid2609.ET2609 